\title{
A CONTROLLED PLUS CONSTRUCTION FOR CRUMPLED LAMINATIONS
}

\author{
R. J. DAVERMAN AND F. C. TINSLEY
}

\begin{abstract}
Given a closed $n$-manifold $M(n>4)$ and a finitely generated perfect subgroup $P$ of $\pi_{1}(M)$, we previously developed a controlled version of Quillen's plus construction, namely a cobordism $(W, M, N)$ with the inclusion $j: N \mapsto W$ a homotopy equivalence and kernel of $i_{\#}: \pi_{1}(M) \mapsto \pi_{1}(W)$ equalling the smallest normal subgroup of $\pi_{1}(M)$ containing $P$ together with a closed map $p: W \mapsto[0,1]$ such that $p^{-1}(t)$ is a closed $n$-manifold for every $t \in[0,1]$ and, in particular, $M=p^{-1}(0)$ and $N=p^{-1}(1)$. We accomplished this by constructing an acyclic map of manifolds $f: M \mapsto N$ having the right fundamental groups, and $W$ arose as the mapping cylinder of $f$ with a collar attached along $N$. The main result here presents a condition under which the desired controlled plus construction can still be accomplished in many cases even when $\pi_{1}(M)$ contains no finitely generated perfect subgroups. By-products of these results include a new method for constructing wild embeddings of codimension one manifolds and a better understanding of perfect subgroups of finitely presented groups.
\end{abstract}

\section{INTRODUCTION}

Given a closed $n$-manifold $M(n>4)$ and perfect subgroup $P$ of $\pi_{1}(M)$ whose normal closure in $\pi_{1}(M)$ is finitely generated as a normal subgroup, Quillen's plus construction [Q1] provides a cobordism $(W, M, N)$ where $W$ is a compact $(n+1)$-manifold with boundary $M \cup N$, the inclusion $N \subset$ $W$ is a homotopy equivalence, and $\pi_{1}(N)$ is isomorphic to the quotient of $\pi_{1}(M)$ by the normal closure of $P$ in $\pi_{1}(M)$. For some time in a continuing effort to expand on [Li] and [Da3] we have sought to obtain a somewhat more controlled version of Quillen's construction. The desired control is that of a crumpled lamination on $W$, namely, a closed map $p: W \mapsto[0,1]$ such that $p^{-1}(t)$ is a closed $n$-manifold for every $t \in[0,1]$ and, in particular, $M=p^{-1}(0)$ and $N=p^{-1}(1)$. We often denote a crumpled lamination by the 4-tuple $(W, M, N, p)$. With finitely generated subgroups $P$ the goal was achieved in [DT2, Theorem 5.2], basically through the construction of an acyclic map of manifolds $f: M \mapsto N$ having the right fundamental groups, and $W$ then arose as the mapping cylinder of $f$ together with a collar attached along $N$. However, there do exist manifolds $M$ whose fundamental groups contain

Received by the editors August 6, 1992.

1991 Mathematics Subject Classification. Primary 57N70, 57N15; Secondary 54B15, 57M05, $57 \mathrm{M} 20$.

Key words and phrases. Crumpled lamination, degree one map, almost acyclic, and perfect normal subgroup. 
(non-finitely-generated) perfect subgroups $P$ for which there is no acyclic map from $M$ to another manifold $N$ killing $P$ at the fundamental group level. The main result here presents a condition under which desired controlled plus construction can still be accomplished.

The word 'crumpled' hints at the likelihood of wildness in this sort of lamination $p$ on $(W, M, N)$. Indeed, if the manifolds $M$ and $N$ are homotopically inequivalent, then some $p^{-1}(t)$ must be wildly embedded in $W$ [Da3, Corollary 4.3]. Although the literature on wild and flat embeddings includes an extensive variety of results and examples, little of it readily applies to this particular setting, and a by-product of this paper is a new method for constructing wild embeddings of codimension one manifolds.

We call the unique maximal perfect subgroup of any group, $G$, its wild group, written Wild $(G)[C 2$, p. 859]. For any subset, $S$, of $G$ we denote the normal closure of $S$ in $G$ by $\operatorname{ncl}(S, G)$, and define it to be the smallest normal subgroup of $G$ containing $S$.

The simplest example of a group $G$ for which the methods here provide a crumpled lamination $(W, M, N)$, where $\pi_{1}(M) \cong G$ and $\pi_{1}(N)$ is a proper quotient of $G$, is the group presented as

$$
G=\left\langle y, t \mid y=\left[y, y^{\left(y^{t}\right)}\right]\right\rangle
$$

(here, $y^{t}=t^{-1} y t$ ). Howie $[\mathrm{H}]$ has shown that $G$ has no finitely generated perfect subgroups, so our earlier construction does not apply. Furthermore, Tinsley has shown [T] that every acyclic map $f$ from an arbitrary manifold $M$ with fundamental group $G$ to an ANR $X$ has trivial $\pi_{1}$-kernel. A single Tietze transformation yields the presentation

$$
G=\left\langle y, x, t \mid y=\left[y, y^{x}\right], t x t^{-1}=y\right\rangle .
$$

Let $H$ be the subgroup $\left\langle y, x \mid y=\left[y, y^{x}\right]\right\rangle$ and $K$ the 2-complex arising naturally from the given presentation of $H$. The relevant data are:

(1) For any $n$-manifold $M$ with $n>4$ and $\pi_{1}(M) \cong G$ there is a locally tame embedding $f: K \mapsto M$ with $f_{\#}\left(\pi_{1}(K)\right)=H$.

(2) $\operatorname{Wild}(H)=\operatorname{ncl}(y, H) \neq 1$.

(3) $\operatorname{ncl}(\operatorname{Wild}(H), G)=\operatorname{ncl}(H, G)$.

(4) $H_{1}(K) \cong \mathbb{Z}$ and $H_{2}(K) \cong 0$.

A corollary to our work is the construction of a crumpled lamination $(W, M, N)$ with $\pi_{1}(M) \cong G$ and $\pi_{1}(N) \cong G / H \cong \mathbb{Z}$.

To state a more general theorem, we need a definition. A finite 2-complex $K$ is said to be almost-acyclic if $H_{2}(K ; Z) \cong 0$ and $H_{1}(K ; \mathbb{Z})$ is free. The main result of the paper appears in $\S 2$.

Theorem 2.0. Suppose $K$ is an almost acyclic 2-complex and $f: K \mapsto M$ is a locally tame embedding such that

$$
f_{\#}\left(\pi_{1}(K)\right)<\operatorname{ncl}\left(f_{\#}\left(\operatorname{Wild}\left(\pi_{1}(K)\right)\right), \pi_{1}(M)\right) .
$$

Then there exists a crumpled lamination $(W, M, N, p)$ with $p^{-1}\left(\left[0, \frac{3}{4}\right)\right) \cong$ $M \times[0,1), p^{-1}\left(\left[\frac{3}{4}, 1\right]\right) \cong N \times[0,1]$, and

$$
\pi_{1}(N) \cong \pi_{1}(M) / \operatorname{ncl}\left(\operatorname{image}\left(f_{\#}\right), \pi_{1}(M)\right) .
$$


We use Theorem 5.1 of [DT2] to characterize certain cobordisms up to $h$ cobordism. A cobordism $(W, M, N)$ is a one-sided $h$-cobordism if the inclusion $j: N \mapsto W$ is a homotopy equivalence. A finitely presented group is almost-acyclic if it has a finite presentation whose associated 2-complex is almost acyclic. Then

Theorem 2.2. Suppose $(W, M, N)$ is a one-sided h-cobordism with $\operatorname{ker}\left(j_{\#}\right)$ $<H=\operatorname{ncl}\left(\operatorname{Wild}(H), \pi_{1}(M)\right)$ where $H$ is an almost acyclic subgroup of $\pi_{1}(M)$ and $j: M \mapsto W$ is inclusion. Then $(W, M, N)$ admits a crumpled lamination $p: W \mapsto[0,1]$ with $p^{-1}\left(\left[0, \frac{3}{4}\right)\right) \cong M \times\left[0, \frac{3}{4}\right)$ and $p^{-1}\left(\left[\frac{3}{4}, 1\right]\right)$ an h-cobordism (possibly nontrivial).

Under the hypotheses of Theorem 2.0 we show in $\S 1$ how to construct a degree one map $\Delta: M \mapsto N$ with $\operatorname{ker}\left(\Delta_{\#}\right) \cong \pi_{1}(M) / \operatorname{ncl}\left(\operatorname{image}\left(f_{\#}\right), \pi_{1}(M)\right)$. In this simpler setting we give many of the details necessary for the proof of Theorem 2.0. Section 2 develops relative versions of the results in the previous section necessary for the proof of the main theorem. In $\S 3$ we give the arguments sufficient to shrink the cell-like decompositions which arise at the end of $\S 2$. Other than the technology of Edwards [E2], $\S 3$ is self-contained. Finally, in the spirit of Howie $[\mathrm{H}]$ we further classify perfect subgroups of finitely presented groups. In particular, we introduce two new classes motivated by the geometric and group-theoretic content of this paper and investigate the behavior of these under the standard group theoretic operations.

\section{Almost ACYCLIC COMPLEXES AND DEGREE ONE MAPS}

In this section we show how to construct special degree one maps of manifolds by identifying almost acyclic complexes to points. Our construction also serves as a model for construction of a corresponding crumpled lamination in the next section.

For the remainder of this section let $G$ be a finitely presented group and $M$ a closed, orientable $n$-manifold with $n>4$ and $\pi_{1}(M) \cong G$. We prove the following:

Proposition 1.0. Suppose $K$ is an almost acyclic 2-complex and $f: K \mapsto M$ is a locally tame embedding such that

$$
f_{\#}\left(\pi_{1}(K)\right)<\operatorname{ncl}\left(f_{\#}\left(\operatorname{Wild}\left(\pi_{1}(K)\right)\right), \pi_{1}(M)\right) .
$$

Then there exists a degree one map $\Delta: M \mapsto N$ onto an n-manifold, $N$, such that $\Delta$ equals the composition $\nu \circ \delta \circ h \circ \mu$ where $\mu$ identifies $f(K)$ to a point, $h$ is an approximate inverse to a cell-like map, $\delta$ identifies a wedge of 2-spheres to a point, and $\nu$ is an acyclic map.

A corollary to the construction of $\Delta$ is that

$$
\operatorname{ker}\left(\Delta_{\#}\right)=\operatorname{ncl}\left(f_{\#}\left(\pi_{1}(K)\right), \pi_{1}(M)\right) .
$$

Denote the commutator subgroup of a group $G$ by $[G, G]$. Recall that a group $G$ is perfect if $G=[G, G]$. Finally, a complex is acyclic if its reduced homology with $\mathbb{Z}$-coefficients is trivial.

If $\pi_{1}(M)$ contains a finitely generated perfect subgroup, $P$, then there is an acyclic 2-complex $K$ and a nice embedding $f: K \mapsto M$ with $\pi_{1}(M / f(K)) \cong$ $\pi_{1}(M) / \operatorname{ncl}\left(P, \pi_{1}(M)\right)$ [DT2]. Let $\mu: M \mapsto M / f(K)$ be the decomposition 
map. Now, $M / f(K)$ is a generalized manifold which fails to be a manifold at a single point. $M / f(K)$ has a resolution $d: N \mapsto M / f(K)$ where $N$ is an $n$-manifold and $d$ has a single nontrivial, cell-like point-preimage [CBL] or [Qn2]. For an appropriate approximate inverse $h$ to $d$, the composition $h \circ \mu: M \mapsto N$ is of degree one and $\operatorname{ker}\left((h \circ \mu)_{\#}\right)=\operatorname{ncl}\left(f_{\#}\left(\pi_{1}(K)\right), \pi_{1}(M)\right)$.

We use this construction as a model for building special degree one maps of manifolds where, in fact, $\pi_{1}(M)$ may contain no nontrivial finitely generated perfect subgroups. In particular, the kernel of the induced $\pi_{1}$-homomorphism is not the normal closure of a finitely generated perfect group.

Suppose that $K$ is an almost acyclic 2-complex, $f: K \mapsto M$ is a locally tame embedding, $f_{\#}: \pi_{1}(K) \mapsto \pi_{1}(M)$ is the induced homomorphism on fundamental groups, and that

$$
1 \neq f_{\#}\left(\pi_{1}(K)\right)<\operatorname{ncl}\left(f_{\#}\left(\operatorname{Wild}\left(\pi_{1}(K)\right)\right), \pi_{1}(M)\right) .
$$

Identify a manifold mapping cylinder neighborhood $N_{K}$ of $f(K)$ in $M$ [Qn1, Theorem 3.1.1]. If $M$ is PL, we take this neighborhood to be a regular neighborhood of $f(K)$. Let $\mu: M \mapsto M / f(K)$ be the decomposition map. Since $f(K)$ is a polyhedron, $M / f(K)$ is an ANR of finite type. Now $M / f(K)$ fails to be a manifold only at the point $x=|f(K)|$. However, it also fails to be a generalized manifold at this point. In particular,

$$
H_{2}(M / f(K), M / f(K)-x ; \mathbb{Z}) \cong H_{1}(K) \neq 0 \text {. }
$$

Details of (1.2) are included in the Appendix.

Despite the nonmanifold features, $M / f(K)$ still has a resolution in the sense that there is a nicer space, $Y$, and a cell-like map, $d: Y \mapsto M / f(K)$.

To construct $d$ first observe that

$$
\pi_{1}(M / f(K)) \cong \pi_{1}(M) / \operatorname{ncl}\left(f_{\#}\left(\pi_{1}(K)\right), \pi_{1}(M)\right)
$$

By (1.1) necessarily

$$
\operatorname{ncl}\left(f_{\#}\left(\pi_{1}(K)\right), \pi_{1}(M)\right)=\operatorname{ncl}\left(f_{\#}\left(\operatorname{Wild}\left(\pi_{1}(K)\right)\right), \pi_{1}(M)\right)
$$

Since $\pi_{1}(K)$ is finitely generated, there is a finite collection of elements $y_{i} \in$ $\operatorname{Wild}\left(\pi_{1}(K)\right)$ with

$$
\operatorname{ncl}\left(f_{\#}\left(\pi_{1}(K)\right), \pi_{1}(M)\right)=\operatorname{ncl}\left(\left\{y_{1}, \ldots, y_{t}\right\}, \pi_{1}(M)\right) .
$$

We now obtain the resolution using the technique developed by Cannon [C1] and explicated by Daverman [Da4, Chapter VII]. For each $y_{i}$ there is an open grope, $D_{i}$, and a pointed (with basepoint in bdy $\left(D_{i}\right)$ ) map, $p_{i}: D_{i} \mapsto K$, such that $p_{i}\left(\operatorname{bdy}\left(D_{i}\right)\right)$ is a loop representing $y_{i} \in \mathrm{Wild}\left(\pi_{1}(K)\right)$. Since $f: K \mapsto M$ is locally tame, $p_{i}$ induces a map $\bar{p}_{i}: D_{i}^{+} \mapsto M / f(K)$ where $D_{i}^{+}$is the closed grope, $\bar{p}_{i}\left(D_{i}\right) \subset M / f(K)-x$, and $\bar{p}_{i}\left(D_{i}^{+}-D_{i}\right)=x \quad\left(D_{i}^{+}-D_{i}\right.$ is called the tip set of $\left.D_{i}\right)$. Since $n \geq 5$, we may assume $\bar{p}_{i} \mid D_{i}$ is a locally tame embedding and that the images $\bar{p}_{i}\left(D_{i}\right)$ are mutually disjoint. For each $i, 1 \leq i \leq t$, we can identify a manifold mapping cylinder neighborhood, $P_{i}$, of each $\bar{p}_{i}\left(D_{i}\right) \subset$ $M / f(K)-x$ with $r_{i}: P_{i} \mapsto \bar{p}_{i}\left(D_{i}\right)$ the associated strong deformation retraction satisfying

$$
\lim _{* \mapsto x}\left(\operatorname{diam}\left(r_{i}^{-1} \circ r_{i}(*)\right)\right)=0 \text { uniformly. }
$$


There is a map $\tau_{i}: C_{i}^{n} \mapsto P_{i} \cup\{x\}$ where $C_{i}^{n}$ is a crumpled cube, $\tau_{i}$ is an embedding over $P_{i}$, and $\tau_{i}^{-1}(x)$ is a wild Cantor set $\mathbf{C}_{i}$ in $\operatorname{bdy}\left(C_{i}^{n}\right)=S^{n-1}$.

There exist a $C E$ map $\varsigma_{i}: B_{i}^{n} \mapsto C_{i}^{n}$, where $B_{i}^{n}$ is an $n$-cell, and a collar $\lambda_{i}: S^{n-1} \times[0, \infty) \mapsto B_{i}^{n}$ on $\operatorname{bdy}\left(B_{i}^{n}\right)$ such that (1.6.3) the nontrivial point-preimages of $\varsigma_{i}$ are precisely the wild arcs $\left\{\lambda_{i}(c \times[0,1]) \mid c \in \mathbf{C}_{i}\right\}, \varsigma_{i} \mid \lambda_{i}\left(S^{n-1} \times 0\right)=$ identity, and $\lambda_{i}\left(\mathbf{C}_{i} \times 1\right)$ is tamely embedded in $\operatorname{int}\left(B_{i}^{n}\right)$.

Identify loops $\left\{l_{1}, l_{2}, \ldots, l_{s}\right\}$ in $K$ representing the generators of $H_{1}(K ; \mathbb{Z})$. Homotopically move each $f\left(l_{j}\right)$ off $f(K)$ in $M$ to an embedded loop, $\hat{l}_{j}$, so that the homotopy is an isotopy off $f(K)$ and misses $\mu^{-1}\left(\bigcup_{i=1}^{t} P_{i}\right)$. By (1.5) and general position, $\hat{l}_{j}$ bounds a disk with holes, $\Theta_{j}$, so that

$$
\begin{gathered}
\left(\Theta_{j}-\operatorname{bdy}\left(\Theta_{j}\right)\right) \subset\left(M-f(K)-\mu^{-1}\left(\bigcup_{i=1}^{t} P_{i}\right)\right), \\
\operatorname{bdy}\left(\Theta_{j}\right)-\hat{l}_{j} \subset \mu^{-1}\left(\bigcup_{i=1}^{t} P_{i}\right)
\end{gathered}
$$

with the inclusion an embedding on each component.

Let

$$
Y=\left(M / f(K)-\left(\bigcup_{i=1}^{t} \operatorname{int}\left(P_{i}\right)\right)\right) \cup\left(\bigcup_{i=1}^{t} B_{i}^{n}\right)
$$

where $B_{i}^{n}$ is attached to $\operatorname{bdy}\left(P_{i}\right) \cup\{x\}$ by the map $\tau_{i} \circ \varsigma_{i} \mid S^{n-1}$.

Lemma 1.1. $Y$ is an ANR.

Proof. What distinguishes this setting from that of Cannon is that $M / f(K)$ is not a generalized manifold. However, well-known results would apply if each $\tau_{i}: C_{i}^{n} \mapsto P_{i} \cup\{\dot{x}\}$ were an embedding. We show how to reduce our situation to the case of an embedding for $n \geq 6$. We omit details for the case $n=5$ which requires altering $K$ in the manner discussed at the beginning of the next section.

Without much loss of generality, there is a tame embedding $\gamma: K \times[0,1] \mapsto$ $\operatorname{int}\left(N_{K}\right)$ with $\gamma \mid K \times\{1\}=f$. To see why, use general position to find an embedding $f_{1}: K \mapsto$ bdy $\left(N_{K}\right)$ homotopic to $f$ in $N_{K}$, let $\gamma$ denote the product embedding $f_{1} \times$ id: $K \times[0,1] \mapsto$ bdy $\left(N_{K}\right) \times[0,1]$ into a collar on bdy $\left(N_{K}\right)$, and replace the original $f$ with $\gamma \mid K \times\{1\}$. Let $\Gamma$ be the usc decomposition of $M$ consisting of points together with the nondegenerate elements $\{\gamma(K \times\{t\}) \mid t \in[0,1]\}$. Now we can embed each closed grope, $D_{i}^{+}$, in $M / \Gamma$, by spreading out successive grope stages to different levels of the collar on bdy $\left(N_{K}\right)$. This embeds each tip set in the image of the nondegeneracy set, an arc, $\alpha$. Here $M / \Gamma$ is an ANR (it is a union of more obvious ANRs $\left(M-\operatorname{int}\left(N_{K}\right)\right) / \gamma(K \times 0),\left(\right.$ bdy $\left.\left(N_{K}\right) / \gamma(K \times 0)\right) \times[0,1]$, and a third piece homeomorphic to $\left.N_{K} / \gamma(K \times 0)\right)$. As in [CBL] or [Da4, p. 276], when we take thickenings, $P_{i}^{\prime}$, of the embedded gropes, then

$$
Y^{\prime}=M / \Gamma-\left(\bigcup \operatorname{int}\left(P_{i}^{\prime}\right)\right) \cup\left(\bigcup B_{i}^{n}\right)
$$


is an ANR. Finally, $Y \cong Y^{\prime} / \alpha$ so $Y$ is an ANR [Sm]. This completes the proof of Lemma 1.1.

The construction identifies a cell-like map $d: Y \mapsto M / f(K)$ which has a single nontrivial point-preimage, $d^{-1}(x)$, homeomorphic to a wedge of arcs, $\bigvee_{l \in \mathbf{C}} A_{l}$, where $\mathbf{C}$ is a Cantor set and $A_{l}$ is an arc. Let $y$ be the wedge point. Then the map $d:(Y, y) \mapsto(M / f(K), x)$ induces isomorphisms on local homology. In particular, $Y-y$ is a manifold and

$$
\begin{aligned}
H_{2}(Y, Y-y ; \mathbb{Z}) & \cong H_{2}\left(Y, Y-d^{-1}(x)\right) \cong H_{2}(M / f(K), M / f(K)-x ; \mathbb{Z}) \\
& \cong H_{1}(K ; \mathbb{Z})
\end{aligned}
$$

and each is finitely generated. We discuss this fact also in the Appendix.

Now, let $\kappa_{j}$ be the image under $d^{-1} \circ \mu$ of the homotopy taking $f\left(l_{j}\right)$ to $\hat{l}_{j}$. Then

$$
\boldsymbol{\Theta}_{j}^{\prime}=\left(d^{-1} \circ \mu\left(\boldsymbol{\Theta}_{j}\right) \cup\left(\kappa_{j}-f\left(l_{j}\right)\right) \cup\{y\}\right) \subset\left(\left(Y-d^{-1}(x)\right) \cup\{y\}\right)
$$

is a disk with holes with one fewer boundary components than $\boldsymbol{\Theta}_{j}\left(f\left(l_{j}\right)\right.$ is mapped to $y)$. The previous discussion allows that each boundary component of $\boldsymbol{\Theta}_{j}^{\prime}$ is homotopically trivial in the manifold $(Y-\{y\})$. General position in $(Y-\{y\})$ yields a wedge of 2-spheres, $S=\bigvee_{j=1}^{s} S_{j}^{2}$, locally tame modulo the wedge point $y$ where each $S_{j}^{2}$ arises naturally as $\Theta_{j}^{\prime}$ with disks attached to boundary components. By construction, the inclusion of $S$ into $Y$ induces isomorphisms on homology. In particular,

$$
H_{2}(S ; \mathbb{Z}) \stackrel{\cong}{\longrightarrow} H_{2}(S, S-y ; \mathbb{Z}) \stackrel{\cong}{\longrightarrow} H_{2}(Y, Y-y ; \mathbb{Z}) \cong \mathbb{Z}^{s}
$$

where $\mathbb{Z}^{s}$ denotes the direct sum of $s$ copies of $\mathbb{Z}$. Let $Z=Y / S, \delta: Y \mapsto Z$ be the decomposition map, and $z=\delta(S)$.

Lemma 1.2. $Z$ is a generalized n-manifold.

Proof. A detailed proof appears in the Appendix. However, the idea is as follows. First, since $Y$ and $S$ are both ANRs, so is $Z$. Next, since the 2-complex $\mathrm{K}$ is almost acyclic, then the complex, $\bar{K}$, obtained by abstractly attaching disks to loops representing the generators of $H_{1}(K ; \mathbb{Z})$, is acyclic. So if $\bar{K}$ were embedded in an $n$-manifold, $M$, then $M / \bar{K}$ would be a generalized manifold. Our construction is homologically equivalent to this. We first collapse $K$ obtaining $M / K$. Next we apply the inverse of a cell-like map which does not affect local homology. Finally, we identify the disks attached to representatives of the generators of $H_{1}(K ; \mathbb{Z}$ ) (which at this stage are topologically a wedge of 2-spheres), collapse them out, and, in effect, complete the collapse of $\bar{K}$ in two steps.

Since $Z$ is a manifold except possibly at $z$, then $Z$ is a manifold if given any neighborhood $U$ of $z$ in $Z$ there exists a neighborhood $V$ of $z$ in $Z$ so that any map $g: \operatorname{bdy}(B) \mapsto V-z$ extends to a map $\bar{g}: B \mapsto U-z$ where $B$ is a 2-disk. As in [C1, p. 107], this local property implies $Z$ has the DDP, and then $Z$ is a manifold by [Qn3].

Lemma 1.3. If $\left[\pi_{1}(K), \pi_{1}(K)\right]=\mathrm{Wild}(K)$, then $Z$ is a manifold.

Proof. Let $z \in U^{\text {nbhd }} \subset Z$. Since $Z$ is an ANR, there exist a neighborhood $V$ of $z$ in $U$ and a strong deformation retraction of $V$ to $z$ in $U$. Let 
$g$ : $\operatorname{bdy}(B) \mapsto V$ be a mapping of the boundary of a disk. Since $S$ is simply connected, there is a map $g_{1}: B \mapsto \delta^{-1}(U) \subset Y$ so that $\delta \circ g_{1}|\operatorname{bdy}(B)=g| \operatorname{bdy}(B)$. But $(S-y)$ is locally tame in $(Y-y)$ so we may adjust $g_{1}$ slightly to make $g_{1}(B) \cap(S-y)=\varnothing$. Finally, since $d^{-1}(x)-y$ has embedding codimension 2 in $Y-y$ and $Y$ rather obviously satisfies the disjoint arc-disk property of [Da2], we can adjust slightly $g_{1}$ so $\left(d \circ g_{1}\right)^{-1}(x)$ is 0 -dimensional and $\delta \circ g_{1} \mid \operatorname{bdy}(B)$ is homotopic in $V-z$ to $g \mid \operatorname{bdy}(B)$.

Since $d: Y \mapsto M / f(K)$ is a homeomorphism over $M / f(K)-\operatorname{int}\left(\bigcup P_{i}\right)$, we may choose the manifold mapping cylinder neighborhood, $N_{K}$, of $K$ in $M$ so that $d^{-1}\left(\mu\left(N_{K}\right)-\bigcup P_{i}-x\right) \subset \delta^{-1}(V)$. By (1.6.1) there is a smaller manifold mapping cylinder neighborhood, $N_{K}^{\prime}$, so that for any $(n-3)$-complex in $\mu\left(N_{K}^{\prime}\right)-x$ there is a general position isotopy with support in $\mu\left(N_{K}\right)-x$ which moves that complex into $\left(M / f(K)-x-\bigcup P_{i}\right)$.

For any neighborhood, $U_{1}$ (we emphasize that $U_{1}$ is chosen independent of $U$ and $V$ and may be arbitrarily small) with $y \in U_{1}$, surface topology and the 0-dimensionality of $g_{1}^{-1}(y)$ allow us to identify a disk with holes, $B^{\prime} \subset B-g_{1}^{-1}(y)$, so that $\operatorname{bdy}(B) \subset \operatorname{bdy}\left(B^{\prime}\right)$, and for each component, $\rho B_{k}^{\prime}$, of $\operatorname{bdy}\left(B^{\prime}\right)$ not equal to $\operatorname{bdy}(B), g_{1}\left(\rho B_{k}^{\prime}\right) \subset U_{1}-y$.

We focus on a particular loop $\gamma_{k}=g_{1} \mid \rho B_{k}^{\prime}$. Since $d^{-1}(x)-y$ has embedding codimension 2 in $Y-y$, we may assume image $\left(\gamma_{k}\right) \cap d^{-1}(x)=\varnothing$. Thus, the loop $\gamma_{k}$ is homotopic in $\delta^{-1}(U-z)$ to a loop $\gamma_{k}^{\prime}$ with image in $d^{-1}\left(\mu\left(N_{K}^{\prime}\right)-x-\bigcup P_{i}\right)$. To see this, note first that $\tau_{i} \circ \varsigma_{i}: B_{i}^{n} \mapsto C_{i}^{n} \mapsto P_{i} \cup\{x\}$ is an embedding over image $\left(\gamma_{k}\right) \cap P_{i}$. Then, using the fact that the Cantor set, $\mathbf{C}_{i}$, has embedding codimension 2 in $\operatorname{bdy}\left(B_{i}^{n}\right)$, build a homotopy in $\bigcup B_{i}^{n}$ which fixes $\operatorname{bdy}\left(B_{i}^{n}\right)$ and pushes $\left(\varsigma_{i}^{-1} \circ \tau_{i}^{-1}\left(\operatorname{image}\left(\gamma_{k}\right)\right)\right) \bigcap \operatorname{int}\left(B_{i}^{n}\right)$ along a short collar to $\operatorname{bdy}\left(B_{i}^{n}\right)-\mathbf{C}_{\mathrm{i}}$. For $U_{1}$ sufficiently small, the image under $\tau_{i} \circ \varsigma_{i}$ of the tracks of the homotopy will stay inside $\delta^{-1}(U-z)$ with the final stage in $d^{-1}\left(\mu\left(N_{K}^{\prime}\right)-x-\operatorname{int}\left(\bigcup P_{i}\right)\right)$. But $d^{-1}\left(\bigcup\right.$ bdy $\left.\left(P_{i}\right)\right)$ is locally tame and, thus, collared in $Y-\left(\bigcup \tau_{i} \circ \varsigma_{i}\left(B_{i}^{n}\right)\right)$ so $\gamma_{k}^{\prime}$ results from pushing the final stage off $\left(\bigcup \tau_{i} \circ \varsigma_{i}\left(B_{i}^{n}\right)\right)$ using so little of the collar that image $\left(\gamma_{k}^{\prime}\right)$ remains in $d^{-1} \circ \mu\left(N_{K}^{\prime}\right)$.

Since Wild $\left(\pi_{1}(K)\right)=\left[\pi_{1}(K), \pi_{1}(K)\right]$, we see that

$$
\left\{\operatorname{Wild}\left(\pi_{1}(K)\right),\left|l_{1}\right|, \ldots,\left|l_{s}\right|\right\}
$$

generates $\pi_{1}(K)$. Since $\pi_{1}(K)$ is finitely generated, we may assume that the elements

$$
\left\{y_{1}, \ldots, y_{t},\left|l_{1}\right|, \ldots,\left|l_{s}\right|\right\}
$$

generate $\pi_{1}(K)$ (we may have to enlarge $t$ ).

By regular neighborhood theory,

$$
\mu\left(N_{K}^{\prime}\right)-x \cong \operatorname{bdy}\left(N_{K}^{\prime}\right) \times(0,1]
$$

and each has fundamental group isomorphic to $\pi_{1}(K)$. The previous paragraph shows $d \circ \gamma_{k}^{\prime}$ bounds a disk with holes in $\mu\left(N_{K}^{\prime}\right)-x$ so that each other boundary component represents a composite of elements from $\left\{y_{1}, \ldots, y_{t},\left|l_{1}\right|, \ldots,\left|l_{s}\right|\right\}$.

Let $\beta$ be a loop in $\mu\left(N_{K}^{\prime}\right)-x$ representing a $y_{i}$ and recall the earlier grope construction. For any positive integer $m, y_{i}=\prod_{j=1}^{n_{m}} y_{i j m}$ where $y_{i j m}$ belongs 
to $\left(\pi_{1}(K)\right)^{(m)}$, the $m$ th term in the derived series of $\pi_{1}(K)$, and is represented by a boundary curve in the $m$ th stage of the grope, $D_{i}$. We realize this equality as a disk with holes in $\mu\left(N_{K}^{\prime}\right)-x$ having $\beta$ as one boundary component and each other representing a $y_{i j m}$. After performing this construction for each $\beta$ making up the composite referred to in the previous paragraph and repeating for all boundary components of the disk with holes other than $d \circ \gamma_{k}^{\prime}$, we obtain a larger disk with holes having image $\left(d \circ \gamma_{k}^{\prime}\right)$ as one boundary component and each other boundary component representing either an $\left|l_{j}\right|$ or a $y_{i j m}$. By general position, (1.6.1), and (1.14) we may also assume that each other boundary component lies in $\left(d\left(U_{1}\right)-\bigcup P_{i}\right)$.

We use general position fixing the boundary of the disk with holes and our choice of $N_{K}^{\prime}$ to move the disk with holes into $\left(\mu\left(N_{K}\right)-\bigcup P_{i}-x\right)$. Then $d^{-1}$ maps this back to a disk with holes in $d^{-1}\left(\mu\left(N_{K}\right)-\bigcup P_{i}-x\right)$ with image $\left(\gamma_{k}^{\prime}\right)$ a boundary component. A boundary component representing an $\left|l_{j}\right|$ may be capped off in with a disk close to $\left(S_{j}^{2}-y\right)$. For m sufficiently large a boundary component representing a $y_{i j m}$ may be capped off in $d^{-1}\left(\left(\mu\left(N_{K}\right)-\bigcup P_{i}\right)-S\right) \subset$ $\delta^{-1}(U)-S$. This cap necessarily will hit $d^{-1}(x)-y$.

Putting together all of the disks with holes and corresponding caps yields a map $g_{2}: B \mapsto\left(\delta^{-1}(U)-S\right)$ with $\delta \circ g_{2}|\operatorname{bdy}(B)=g| \operatorname{bdy}(B)$. We take as our map, $\bar{g}=\delta \circ g_{2}$.

This completes the proof of Lemma 1.3.

Lemma 1.4. If $\operatorname{Wild}\left(\pi_{1}(K)\right)=\operatorname{ncl}\left(\left\{y_{1}, \ldots, y_{m}\right\}, \pi_{1}(K)\right)$, then there is an almost acyclic 2-complex, $K^{\prime}$, and a locally tame embedding $f^{\prime}: K^{\prime} \mapsto M$ satisfying the hypotheses of Lemma 1.3, with

$$
\operatorname{ncl}\left(f_{\#}^{\prime}\left(\pi_{1}\left(K^{\prime}\right)\right), \pi_{1}(M)\right) \cong \operatorname{ncl}\left(f_{\#}\left(\pi_{1}(K)\right), \pi_{1}(M)\right) .
$$

Proof. For each $y_{i} \in \operatorname{Wild}\left(\pi_{1}(K)\right)$, necessarily $y_{i}=\prod_{j=1}^{n_{i}}\left[u_{i j}, v_{i j}\right]$ where $u_{i j}, v_{i j} \in \operatorname{Wild}\left(\pi_{1}(K)\right)$. We begin the construction of $K^{\prime}$ with a bouquet of $m$ simple closed curves, one for each $y_{i}$. To the $i$ th simple closed curve we attach a disk with $n_{i}$ handles representing the relation, $y_{i}=\prod_{j=1}^{n_{i}}\left[u_{i j}, v_{i j}\right]$. Finally, since each $u_{i j}$ (respectively $v_{i j}$ ) is in $\operatorname{ncl}\left(\left\{y_{1}, \ldots, y_{m}\right\}, \pi_{1}(K)\right.$ ), we attach a disk with holes with one boundary component attached to the meridian (respectively longitude) of handle $(i, j)$ and each other boundary component attached to one of the original simple closed curves. Since all of the relations above are relations in $\pi_{1}(K)$, the resulting complex, $K^{\prime}$, embeds in a regular neighborhood of $f(K)$ in $M$ with the image of the ith simple closed curve of $K^{\prime(1)}$ representing $y_{i} \in \pi_{1}(K)$. It is a straightforward exercise to show that $K^{\prime}$ is almost acyclic with $H_{1}\left(K^{\prime} ; \mathbb{Z}\right)$ having a generator for each boundary of each disk with holes which attaches to a $\left|y_{i}\right|$ and that $\operatorname{Wild}\left(\pi_{1}\left(K^{\prime}\right)=\left[\pi_{1}\left(K^{\prime}\right), \pi_{1}\left(K^{\prime}\right)\right]\right.$.

Lemma 1.5. If neither the hypotheses of Lemma 1.3 nor Lemma 1.4 hold, then there is an acyclic map $\nu: Z \mapsto N$ where $N$ is an $n$-manifold and $\delta \circ d^{-1} \circ \mu$ is an embedding over $S(\nu)$. the singular set of $\nu$.

Currently, no known examples fail to satisfy the hypotheses of Lemma 1.4. The question concerning the necessity of Lemma 1.5 is related to a problem raised by Wall [W, Problem F10, p. 387].

Proof. A slight modification to the proof of Lemma 1.1 yields a map $F: K \times$ $[0,1] \mapsto M$ so that $F \mid K \times\{1\}=f$ and $F \mid\left(K^{(1)} \times[0,1] \cup\left(\bigcup K \times t_{k}\right)\right)$ is an 
embedding into $\left(M-\mu^{-1}\left(\bigcup P_{i}\right)-\mu^{-1} \circ d(S)\right)$ for a sequence $\left\{t_{k}\right\}$ increasing to 1 . For each representative $l_{j}$ of a generator of $H_{1}(K ; \mathbb{Z})$ and each $t_{k}$, $d^{-1} \circ \mu \circ F\left(l_{j} \times t_{k}\right)$ is a loop in $Y-S$ which bounds a disk in $Y-S$ close to $S_{j}^{2}$. Let $L_{k}$ be a 2-complex in $Y-S$ obtained by attaching disks to each such generator so that $\left\{\delta\left(L_{k}\right)\right\}$ is a mutally disjoint, null sequence of acyclic 2complexes in $Z-z$. Let $\left\{N_{L_{k}}\right\}_{k=1}^{\infty}$ be a mutually disjoint sequence of manifold mapping cylinder neighborhoods of the $\left\{L_{k}\right\}$ 's in $Z-z$.

Each $N_{L_{k}}$ is a homology cell. Daverman and Tinsley [DT2, Corollary 4.5] show how to construct an acyclic map, $\nu_{k}: N_{L_{k}} \mapsto \mathscr{C}_{k}$, where $\nu_{k}$ is the identity on the boundary, $\nu_{k}$ is an embedding over the complement of a Cantor set, and $\mathscr{C}_{k}$ is a contractible manifold. Let $\nu: Z \mapsto N$ be the map which is the identity on $Z-\left(\bigcup N_{L_{k}}\right)$ and equal to $\nu_{k}$ on $N_{L_{k}}$ and $N$ has the identification topology induced by $\nu$.

Thus, $N$ is a manifold except at possibly $\nu(z)$. Let $W$ be any neighborhood of $\nu(z)$ in $N$. Let $U=\nu^{-1}(W)$. We now argue as in Lemma 1.3 except we choose $V$ to be $\nu$-saturated and work entirely over $Z-\left(U N_{L_{i}}\right)$. The latter is possible by general position since each $N_{L_{k}}$ has a 2-spine. We proceed as before until obtaining the loop $\gamma_{k}^{\prime}$. The loop, $d \circ \gamma_{k}^{\prime}$, is homotopic in $\left(\mu\left(N_{K}\right)-x-\bigcup P_{i}\right)$ to a loop $\gamma_{k}^{\prime \prime}$ in an appropriate $N_{L_{i}}$ close to $f(K)$. The loop $\nu \circ \gamma_{k}^{\prime \prime}$ bounds a disk in $\mathscr{C}_{i}$. We map everything into $N-\nu(z)$ and see that $N$ is an $n$-manifold and the proof of Lemma 1.5 is complete.

Proof of Proposition 1.0. For an appropriate approximate inverse, $h: M / f(K)$ $\mapsto Y$, to $d, \Delta=\delta \circ h \circ \mu: M \mapsto N$ (Lemma 1.3 or Lemma 1.4) or $\Delta=$ $\nu \circ \delta \circ h \circ \mu: M \mapsto N$ (Lemma 1.5) is the desired degree one map.

\section{Crumpled laminations}

We prove the main theorem of this paper.

Theorem 2.0. Suppose $K$ is an almost acyclic 2-complex and $f: K \mapsto M$ is a locally tame embedding such that

$$
f_{\#}\left(\pi_{1}(K)\right)<\operatorname{ncl}\left(f_{\#}\left(\operatorname{Wild}\left(\pi_{1}(K)\right), \pi_{1}(M)\right) .\right.
$$

Then there exists a crumpled lamination $(W, M, N, p)$ with $p^{-1}\left(\left[0, \frac{3}{4}\right)\right) \cong$ $M \times[0,1), p^{-1}\left(\left[\frac{3}{4}, 1\right]\right) \cong N \times[0,1]$, and

$$
\pi_{1}(N) \cong \pi_{1}(M) / \operatorname{ncl}\left(\operatorname{image}\left(f_{\#}\right), \pi_{1}(M)\right) .
$$

The construction begins with $M \times[-1,1]$. Assume $f: K \mapsto M$ is a locally tame embedding of $K$ in $M$ which satisfies (1.1)-(1.5). We adjust $f$ slightly as follows. Let $Q$ be the flat boundary of a topological regular neighborhood $N_{K}$ of $f(K)$ in $M$. Then $f$ is homotopic in this neighborhood to $f_{1}: K \mapsto Q$ where $f_{1}$ is either an embedding $(n>5)$ or an immersion $(n=5)$ with, at worst, double points. In the latter case $f_{1}(K)$ is still an almost acyclic compactum since it has the same homotopy type as $K$ wedged with a bouquet of circles (one circle for each pair of double points). Also, $f_{1}$ also satisfies

$$
\operatorname{ncl}\left(f_{1 \#}\left(\pi_{1}(K)\right), \pi_{1}(M)\right) \cong \operatorname{ncl}\left(f_{\#}\left(\pi_{1}(K)\right), \pi_{1}(M)\right) .
$$

So we take $f_{1}$ for $f$ and, since $Q$ is flat in $M$, we assume

$$
f(K) \subset Q \cong Q \times\{0\} \subset Q \times(-\infty, \infty) \subset M \cong M \times\{0\} \subset M \times[-1,1] .
$$


By the spin of a space $B \times[0, \infty)$ we mean the quotient space obtained from $B \times[0, \infty) \times S^{1}$ by identifying all circles of the form $b \times 0 \times S^{1}, b \in B$. The spin structure provides generalized polar coordinates $\langle b, r, \theta\rangle$ for $B \times \mathbb{R}^{2}$ where $r \in[0, \infty)$ and $\theta \in S^{1}$. For ease of notation we assume the $S^{1}$ coordinate is a complex number (shown in bold) of modulus 1 and that the polar subspace, $b \times[0, \infty) \times\{-1,1\} \quad\left(b \times 0 \times-1 \equiv b \times 0 \times \mathbf{1}, 1 \in S^{1}\right.$, of course $)$, naturally corresponds to $B \times \mathbb{R}^{1} \times 0 \subset B \times \mathbb{R}^{2}$.

In our setting we give the neighborhood

$$
(Q \times(-\infty, \infty)) \times(-\epsilon, \epsilon) \subset M \times[-1,1]
$$

coordinates $\langle q, r, \theta\rangle$ by spinning $Q \times[0, \infty)$ in $M \times(-1,1) \cong M \times(-\infty, \infty)$ and assume

$$
Q \times[0, \infty) \times\{-\mathbf{1}, \mathbf{1}\} \subset M \times 0 \subset M \times[-1,1] .
$$

We will refer to

$$
Q \times 0 \times S^{1} /\left\{q \times 0 \times S^{1} \mid q \in Q\right\} \subset Q \times[0, \infty) \times S^{1} /\left\{q \times 0 \times S^{1} \mid q \in Q\right\}
$$

simply as $Q$.

We will now modify the construction of $\S 1$. By (2.1) the same finite collection of elements $y_{i} \in \operatorname{Wild}\left(\pi_{1}(K)\right)$ satisfies

$$
\operatorname{ncl}\left(f_{\#}\left(\pi_{1}(K)\right), \pi_{1}(M)\right)=\operatorname{ncl}\left(\left\{y_{1}, \ldots, y_{t}\right\}, \pi_{1}(M)\right) .
$$

We mod out $f(K)$ and let

$$
x=|f(K)| \in Q / f(K) \subset M \times 0 / f(K) \subset M \times[-1,1] / f(K)
$$

with $\mu: M \times[-1,1] \mapsto M \times[-1,1] / f(K)$ the decomposition map. As in $\S 1$ we obtain a map $\bar{p}_{i}: D_{i}^{+} \mapsto Q \times[0, \infty) / f(K)$ where $D_{i}^{+}$is a closed grope, $\bar{p}_{i}\left(D_{i}^{+}-D_{i}\right)=x, \bar{p}_{i} \mid D_{i}$ is a locally tame embedding, $\bar{p}_{i}\left(\operatorname{bdy}\left(D_{i}\right)\right)$ is a loop representing $y_{i} \in G$, and so the $D_{i}$ 's have mutually disjoint pinched regular neighborhoods $P_{i} \subset Q \times(0, \infty)$. As before we remove the interior of each $P_{i}$ and attach a cell, $B_{i}^{n}$, via a map

$$
\left(\tau_{i} \circ \varsigma_{i} \mid \operatorname{bdy}\left(B_{i}^{n}\right)\right): \operatorname{bdy}\left(B_{i}^{n}\right) \mapsto \operatorname{bdy}\left(P_{i}\right) \cup x
$$

satisfying (1.6.1)-(1.6.3). Now, simultaneously we make this replacement for each $\theta \in S^{1}$ and denote by $Q_{\theta}^{+}$the space

$$
\left(\left(Q \times(0, \infty)-\left(\bigcup_{i=1}^{t} \operatorname{int}\left(P_{i}\right)\right)\right) \cup\left(\bigcup_{i=1}^{t} B_{i}^{n}\right)\right) \times \theta .
$$

We obtain a space, $\mathscr{Y}$, and a cell-like map $d: \mathscr{Y} \mapsto M \times[-1,1] / f(K)$ where $d$ has a single nontrivial point-preimage, $d^{-1}(x)$, homeomorphic to a wedge of 2-disks, $\bigvee_{\alpha \in \mathbf{C}} B_{\alpha}^{2}$ ( C a Cantor set), with wedgepoint $y$. The Cantor set, $\mathbf{C}$, arises naturally as $\mathbf{C}=\bigcup_{i=1}^{t} \mathbf{C}_{\mathrm{i}}$ where $\mathbf{C}_{i}=\tau_{i}^{-1}(x) \subset C_{i}^{n}$. There is a natural subspace $Y \subset \mathscr{Y}$ so that $(d \mid Y): Y \mapsto M \times 0 / f(K)$ is a cell-like map with single nontrivial point-preimage $(d \mid Y)^{-1}(x)$ homeomorphic to a wedge of arcs, $\bigvee_{\alpha \in \mathrm{C}} A_{\alpha}$, where $A_{\alpha}=B_{\alpha}^{2} \cap Y$. There is a natural projection map $e_{\alpha}: B_{\alpha}^{2} \mapsto A_{\alpha} \quad\left(\right.$ not unlike the map $\left.B^{2} \stackrel{(r, s) \mapsto r}{\longrightarrow} B^{1}\right)$. In particular, we may assume

$$
e_{\alpha}^{-1}(y)-y \subset Q_{\mathbf{i}}^{+} \quad \text { where } \mathbf{i} \in S^{1}
$$


and $Q_{i}^{+}$lies in the same component of $\mathscr{Y}-Y$ as does $d^{-1} \circ \mu(M \times\{-1\})$.

The space $Y$ of this section is quite similar to the $Y$ of $\S 1$. It is an ANR which is a manifold except at $y$ and has the same local homology at $y$. The only difference is that there are two gropes used in this section (corresponding to $-1,1 \in S^{1}$ ) for each grope in $\S 1$. The extra gropes affect neither the local homology nor the ANR status of $Y$.

As before, we construct a wedge of 2-spheres, $S=\bigvee_{j=1}^{s} S_{j}^{2}$ with wedgepoint $y$, in $Y$ so that the inclusion of $S-y$ into $Y-y$ is locally tame and,

$$
H_{2}(S ; \mathbb{Z}) \stackrel{\cong}{\longrightarrow} H_{2}(S, S-y ; \mathbb{Z}) \stackrel{\cong}{\longrightarrow} H_{2}(Y, Y-y ; \mathbb{Z}) \cong \mathbb{Z}^{s} .
$$

But in addition,

$$
H_{2}(S ; \mathbb{Z}) \stackrel{\cong}{\longrightarrow} H_{2}(S, S-y ; \mathbb{Z}) \stackrel{\cong}{\longrightarrow} H_{2}(\mathscr{Y}, \mathscr{Y}-y ; \mathbb{Z}) \cong \mathbb{Z}^{s} .
$$

We identify an usc cell-like decomposition of $\mathscr{Y}$. Denote by $\mathscr{Y}^{+}$the closure of the component of $\mathscr{Y}-Y$ containing $Q_{i}^{+}$. Let $\left(D_{\alpha}, A_{\alpha}\right) \subset(\mathscr{Y}, Y)$ be one of the (disk,arc) pairs constructed above. Denote by $\mathscr{G}^{+}$the usc decomposition of $\mathscr{Y}$ consisting of points, special element $g_{0}=\left\{\left(\bigcup e_{\alpha}^{-1}(y)\right) \cap \mathscr{Y}^{+}\right\}$, and arcs $\left\{e_{\alpha}^{-1}(a) \mid a \in\left(A_{\alpha}-y\right), \alpha \in \mathbf{C}\right\}$.

Lemma 2.1. The decomposition $\mathscr{G}^{+}$is shrinkable.

Proof of Lemma 2.1. We first shrink $g_{0}$ and then show how to apply Corollary 3.4 of the next section. But $g_{0}-y \cong \mathbf{C} \times(0,1]$ is contained in the locally tame codimension one submanifold $Q_{i}^{+}$of $\mathscr{y}-y$ so $g_{0}-y$ is standardly embedded in $\mathscr{Y}^{+}$. By fairly standard 'radial' shrinking methods first introduced by Bing [B or Da4, §8], $g_{0}$ can be shrunk to the point $y$ by a pseudo-isotopy supported in an arbitrary neighborhood of $g_{0}-y$. If $\pi_{g_{0}}: \mathscr{Y} \mapsto \mathscr{Y} / g_{0}$ is the identification map, then we have a homeomorphism $h_{1}: \mathscr{Y} \mapsto \mathscr{Y} / g_{0}$ with $h_{1}\left|Y=\pi_{g_{0}}\right| Y$.

We now apply Corollary 3.4 of the next section. For each $i, 1 \leq i \leq t$, let $U_{i}$ be an open neighborhood of $\pi_{g_{0}}\left(\operatorname{int}\left(B_{i}^{n}\right)\right)$ in $\mathscr{Y} / g_{0}$ so that

$$
\begin{gathered}
U_{i} \cap U_{j}=\varnothing \quad \text { for } i \neq j, \\
\operatorname{clos}\left(U_{i}\right)=\pi_{g_{0}}\left(\operatorname{bdy}\left(P_{i}\right) \cup x\right) .
\end{gathered}
$$

For appropriate saturated neighborhoods, $\mathscr{U}_{i}$, and after possible slight adjustments of the 2-manifold, $T=\pi_{g_{0}}(S-y)$, we piece together a homeomorphism $h_{2}: \mathscr{Y} / g_{0} \mapsto \mathscr{Y} / \mathscr{G}^{+}$so that the composition $h=h_{2} \circ h_{1}: \mathscr{Y} \mapsto \mathscr{Y} / \mathscr{G}^{+}$satisfies $h\left|S=\pi_{\mathscr{G}+}\right| S$. We let the triple

$$
(\mathscr{Z}, Z, z)=\left(\pi_{\mathscr{G}+}(\mathscr{Y}) / h(S), \pi_{\mathscr{G}_{+}}(Y) / h(S), h(y)\right)
$$

and $(\bar{\delta}, \delta):(\mathscr{Y}, Y) \mapsto(\mathscr{Z}, Z)$ denote the decomposition map. We observe that the pair $(\mathscr{Z}, Z)$ is a generalized manifold pair which is a manifold pair except possibly at $z$ and that $\mathscr{Z}^{+}=\left(\bar{\delta}\left(\mathscr{Y}^{+}\right)-Z\right)$ is homeomorphic to $M \times$ $\left[0, \frac{3}{4}\right)$.

We repeat the analysis of $\S 1$. If the hypotheses of either Lemma 1.3 or 1.4 applies, then $Z$ is a manifold, and we let $(W, N)=(\mathscr{Z}, Z)$. If Lemma 1.5 applies, the resulting acyclic map $\nu: Z \mapsto N$ trivially extends to an acyclic map $\bar{\zeta}: \mathscr{Z} \mapsto W$ with the same singular set. In any event, the resulting pair, $(W, N)$, is a manifold pair since the point, $\bar{\zeta}(z)$, is 1-LCC embedded in $W$. 
If Lemma 1.5 and $\nu$ were indeed necessary, $N$ may not be 1-LCC embedded in $\left(W-\mathscr{Z}^{+}\right)$. In this case, we may reembed $\mathscr{Z}^{+} \cup N$ in $W$ so that $N$ has a collar in $\left(W-\mathscr{Z}^{+}\right)$homeomorphic to $N \times\left[\frac{3}{4}, 1\right]$ [Da1]. Then there is an obvious crumpled lamination map $p: \mathscr{Z}^{+} \cup N \times\left[\frac{3}{4}, 1\right] \mapsto[0,1]$ satisfying the conclusion of Theorem 2.0.

\section{DeCOMPOSITION THEORY}

We summarize the decomposition theory necessary to complete the crumpled lamination constructed in $\S 2$.

Let $\lambda: S^{n-1} \times[0,1] \rightarrow S^{n-1} \times[0, \infty)$ be a collar on the boundary; that is, $\lambda$ is an embedding with $\lambda(s, 0)=(s, 0)$ for all $s \in S^{n-1}$. In this setting we definitely do not assume that $\lambda\left(S^{n-1} \times 1\right)$ is bicollared in the image. Suppose $C$ is a fixed compact subset of $S^{n-1}$. The spin (recall §2) $S^{n-1} \times R^{2}$ of $S^{n-1} \times$ $[0, \infty)$ contains a distinguished space

$$
S p(\lambda, C)=\left(\lambda(C \times I) \times S^{1} / \sim\right) \cong\left(C \times I \times S^{1} / \sim\right) \cong C \times B^{2},
$$

given by the obvious identification. We denote $S p\left(\lambda, S^{n-1}\right)$ more simply as $S p(\lambda)$ and note that in these representations $S p(\lambda, C) \subset S p(\lambda)$ corresponds to $C \times B^{2} \subset S^{n-1} \times B^{2}$. The projection $e: B^{2} \rightarrow B^{1}$ sending $(x, y) \in B^{2}$ to $x \in B^{1}$ induces what we call the vertical arc decomposition $\mathscr{G}(\lambda, C)$ of $S^{n-1} \times R^{2}$ associated with $\lambda$ and $C$; it consists of the point preimages under the associated map Id $\times e: C \times B^{2} \rightarrow C \times B^{1}$ together with the singletons from outside $S p(\lambda, C)$.

Theorem 3.1. Suppose $\lambda: S^{n-1} \times[0,1] \rightarrow S^{n-1} \times[0, \infty)$ is a collar on the boundary, $C$ is a compact $k$-dimensional subset of $S^{n-1}$ such that $\lambda(C \times 1)$ is 1-LCC embedded in $S^{n-1} \times[0, \infty), n \geq 4$, and $k \leq n-3$. Then the decomposition map $S^{n-1} \times R^{2} \rightarrow S^{n-1} \times R^{2} / \mathscr{G}(\lambda, C)$ is a near-homeomorphism.

Proof. In this setting Edwards's Cell-like Approximation Theorem [E2] establishes that it suffices to show $S^{n-1} \times R^{2} / \mathscr{G}(\lambda, C)$ has the DDP. Since any two mappings $\mu_{1}, \mu_{2}$ of $B^{2}$ to the quotient space can be approximated by images of lifts $\psi_{1}, \psi_{2}: B^{2} \rightarrow S^{n-1} \times R^{2}$, it is enough to show how to approximate $\psi_{1}, \psi_{2}$ by mappings $\psi_{1}^{\prime}, \psi_{2}^{\prime}$, respectively, such that no element of $\mathscr{G}(\lambda, C)$ meets both $\psi_{1}\left(B^{2}\right)$ and $\psi_{2}\left(B^{2}\right)$. The 1-LCC condition on $\lambda(C \times 1) \subset S^{n-1} \times[0, \infty)$ implies that the inclusion

$$
\lambda(C \times 1) \times S^{1} \subset\left(S^{n-1} \times R^{2}\right) \backslash\left(S^{n-1} \times\{\text { origin }\}\right) \cong S^{n-1} \times(0, \infty) \times S^{1}
$$

is a 1-LCC embedding. Hence, $\psi_{1}, \psi_{2}$ each can be approximated so as to avoid $\lambda(C \times 1) \times S^{1} \subset C \times \operatorname{bdy}\left(B^{2}\right) \subset \partial(S p(\lambda))$. We shall further modify these adjusted images in $S p(\lambda)$, and for this next operation we refer to the projection map

$$
e^{\prime}=\operatorname{Id} \times e: S^{n-1} \times B^{2} \rightarrow S^{n-1} \times B^{1} .
$$

We approximate $\psi_{i}$ by maps $\psi_{i}^{\prime}(i=1,2)$ such that $\psi_{i}, \psi_{i}^{\prime}$ agree outside $\psi_{i}^{-1}\left(S^{n-1} \times \operatorname{Int} B^{2}\right)$ and

$$
e^{\prime} \psi_{1}^{\prime}\left(\psi_{1}^{-1}\left(S^{n-1} \times \operatorname{Int} B^{2}\right)\right) \cap e^{\prime} \psi_{2}^{\prime}\left(\psi_{2}^{-1}\left(S^{n-1} \times \operatorname{Int} B^{2}\right)\right) \cap C \times B^{1}=\varnothing .
$$

For $n \geq 5$ this is simple general position; for $n=4$ it still can be accomplished by first making the relevant images $e^{\prime} \psi_{1}^{\prime}$ and $e^{\prime} \psi_{2}^{\prime}$ intersect in a locally finite 
set in $S^{n-1} \times \operatorname{Int} B^{1}$ and then, with a controlled ambient homeomorphism of $S^{n-1} \times \operatorname{Int} B^{1}$, slipping this intersection off $C \times B^{1}$, which is nowhere dense in $S^{n-1} \times \operatorname{Int} B^{1}$.

Corollary 3.2. Under the hypotheses of Theorem 3.1, suppose $\mathscr{G}$ is another upper semicontinuous decomposition of $S^{n-1} \times R^{2}$ into points and arcs, where every arc from $\mathscr{G}$ is a subarc of one in $\mathscr{G}(\lambda, C)$. Then the decomposition map $S^{n-1} \times$ $R^{2} \rightarrow S^{n-1} \times R^{2} / \mathscr{G}$ is also a near-homeomorphism.

This follows directly from the proof of Theorem 3.1.

In particular, we will exploit a related upper half decomposition $\mathscr{G}^{+}$, nondegenerate elements of which are the intersections of those from $\mathscr{G}(\lambda, C)$ with $C \times B_{+}^{2} \subset C \times B^{2}$, where, of course, $B_{+}^{2}=\left\{(x, y) \in B^{2} \mid y \geq 0\right\}$. Let $\pi^{+}: S^{n-1} \times R^{2} \rightarrow S^{n-1} \times R^{2} / \mathscr{G}^{+}$denote the associated decomposition map. We will need the following relative version of Corollary 3.2.

Theorem 3.3. Suppose $\lambda: S^{n-1} \times[0,1] \rightarrow S^{n-1} \times[0, \infty)$ is a collar on the boundary, $n \geq 4$, and $C$ is a compact 0 -dimensional subset of $S^{n-1}$ such that $\lambda(C \times 1)$ is $1-\mathrm{LCC}$ embedded in $S^{n-1} \times[0, \infty)$. Suppose that, in addition, $\eta: T \rightarrow \operatorname{Int}\left(\operatorname{Sp}(\lambda) \cap\left(S^{n-1} \times B^{1}\right)\right.$ is a locally flat, closed embedding of a 2-manifold $T$. Then $\eta$ can be approximated, arbitrarily closely, by another embedding $\eta^{\prime}$ : $T \rightarrow S^{n-1} \times \operatorname{Int} B^{1}$ such that for every $\mathscr{G}^{+}$- saturated open cover $\mathscr{U}$ of $S^{n-1} \times$ $R^{2}$ there exists a homeomorphism $h: S^{n-1} \times R^{2} \rightarrow S^{n-1} \times R^{2} / \mathscr{G}^{+}$satisfying $h\left|\eta^{\prime}(T)=\pi^{+}\right| \eta^{\prime}(T)$ and $h$ is $\pi^{+}(\mathscr{U})$-close to $\pi^{+}$.

Proof. The key is to approximate $\eta$, in a fashion comparable to that of Theorem 3.1, to make the projection $q$ of $\eta^{\prime}(T) \cap \operatorname{Int}\left(S p(\lambda) \cap\left(S^{n-1} \times B^{1}\right)\right.$ to $S^{n-1}$ have $q \circ \eta^{\prime}(T) \cap C$ tamely embedded in $S^{n-1}$. All this entails is a controlled PL approximation so that, for each simplex $\sigma$ of some triangulation of $T$, $q \circ \eta^{\prime}(\sigma)$ is a simplex in $S^{n-1}$. This forces the 0-dimensional set $q \circ \eta^{\prime}(T) \cap C$ to be standardly embedded in $\lambda\left(S^{n-1} \times[0,1)\right)$. It follows as in [Da4, §28] that $S p\left(\lambda, q \circ \eta^{\prime}(T) \cap C\right) \cong\left(q \circ \eta^{\prime}(T) \cap C\right) \times B^{2}$ is 1 -LCC embedded in $S^{n-1} \times R^{2}$. This means that we can regard $\left(q \circ \eta^{\prime}(T) \cap C\right) \times B^{2}$ as the collection of disks corresponding to $C^{\prime} \times B^{2} \rightarrow S^{n-1} \times R^{2}$, where $C^{\prime} \subset S^{n-1}$ is a standardly embedded 0-dimensional subset. Here it is easy to produce a map $f$ of $S^{n-1} \times R^{2}$ to itself such that

$$
f \mid \eta^{\prime}(T)=\text { identity }
$$

$f$ is one-to-one over $S^{n-1} \times R^{2} \backslash \eta^{\prime}(T)$,

and

$$
f \text { is } \mathscr{U} \text {-close to the identity, }
$$

Now the cell-like map $\pi^{+} \circ f^{-1}: S^{n-1} \times R^{2} \rightarrow S^{n-1} \times R^{2} / \mathscr{G}+$ is $1-1$ over $\pi^{+}$。 $f^{-1} \eta^{\prime}(T)$. Since $\left(S^{n-1} \times R^{2} / \mathscr{G}^{+}\right) \backslash \pi^{+} \circ f^{-1}\left(n^{\prime}(T) \cap S p(\lambda, C)\right)$ is a manifold, it is a direct consequence of Edwards's work [E2] that $\pi^{+} \circ f^{-1}$ can be approximated by a homeomorphism $h: S^{n-1} \times R^{2} \rightarrow S^{n-1} \times R^{2} / \mathscr{G}^{+}$subject to the conditions required in the theorem.

The following corollary necessary in $\S 2$ follows trivially. 
Corollary 3.4. Theorem 3.3 holds if $S^{n-1} \times \mathbb{R}^{2}$ is replaced by $U$ where $U$ is any $\pi^{+}$-saturated open subset of $S^{n-1} \times \mathbb{R}^{2}$.

\section{Perfect SUbGroups of FINITELy PRESENTED GROUPS}

The study of crumpled laminations leads us directly to the study of perfect subgroups of finitely presented groups since whenever $(W, M, N, p)$ is a crumpled lamination, necessarily $\operatorname{ker}\left(i_{\#}\right)$ is perfect where $i: M \mapsto W$ is inclusion [DT2, Lemma 2.5].

In $[\mathrm{H}]$, Howie classifies countable groups according to properties of their perfect subgroups. We warn the reader that these definitions are given as negations of particular properties and, as such, may be somewhat confusing. However, these are convenient for stating the behavior of the classes under the standard group theoretic operations. All groups are countable.

Definition 4.1. A group $G$ belongs to $\mathscr{U}$ if $G$ contains no nontrivial finitely generated perfect subgroups.

Definition 4.2. A group $G$ belongs to $\mathscr{F}$ if $G$ contains no nontrivial perfect subgroups.

Those groups which belong to $\mathscr{F}$ are precisely the transfinite metaabelian groups. Obviously, $\mathscr{F} \subset \mathscr{U}$. We introduce and investigate two intermediate classes of groups. These arise naturally out of the study of crumpled laminations.

Definition 4.3. A group $G$ belongs to $\mathscr{L}$ if whenever $(W, M, N, p)$ is a crumpled lamination, $i: M \mapsto W$ is inclusion, and $\pi_{1}(M)=G$, then necessarily $\operatorname{ker}\left(i_{\#}\right)=1$.

Consequently, crumpled laminations whose elements have fundamental groups belonging to $\mathscr{L}$ are trivial in the sense that the manifolds, $W$, involved are either products or nontrivial $h$-cobordisms [DT2, Theorem 5.1].

By the remark opening this section, $\mathscr{F} \subset \mathscr{L}$. For any finitely presented group $G$ with nontrivial finitely generated perfect subgroup $P$, Daverman and Tinsley exhibit a crumpled lamination $(W, M, N, p)$ with $\pi_{1}(M) \cong G$, $\pi_{1}(N) \cong G /\langle P\rangle$, and $\operatorname{ker}\left(i_{\#}\right) \cong \operatorname{ncl}(P, G)$. As a result, $\mathscr{L} \subset \mathscr{U}$ and, thus, $\mathscr{F} \subset \mathscr{L} \subset \mathscr{U}$. In fact, $W$ is constructed as the union of the mapping cylinder of an acyclic map on $M$ and a possibly nontrivial $h$-cobordism. The nontrivial point preimages of the acyclic map may be chosen to be acyclic 2-complexes.

In this paper we have constructed a crumpled lamination which is fundamentally different. In particular, $\operatorname{ker}\left(i_{\#}\right)$ is not the normal closure of a finitely generated perfect group and $W$ does not have a mapping cylinder structure of the type described above [T].

Definition 4.4. A group $G$ belongs to $\mathscr{A}$ if for every almost acyclic group, $H$, and homomorphism, $\phi: H \mapsto G$, with

$$
\phi(H)<\operatorname{ncl}(\phi(\operatorname{Wild}(H)), G)
$$

it is necessarily the case that $\phi(H)=1$.

Since acyclic 2 -complexes are trivially almost acyclic, we have $\mathscr{A} \subset \mathscr{U}$. In Theorem 2.0 we showed that $\mathscr{L} \subset \mathscr{A}$, so $\mathscr{F} \subset \mathscr{L} \subset \mathscr{A} \subset \mathscr{U}$. 
Our efforts to find interesting examples of crumpled laminations has focused on the following question.

Question 4.5. Which of these containments are proper?

In $[\mathrm{H}]$ Howie shows that the containment $\mathscr{F} \subset \mathscr{U}$ is proper. In particular, Adams's group [Ad],

$$
A=\left\langle y, t \mid y=y^{-1} t^{-1} y^{-1} t y t^{-1} y t\right\rangle
$$

belongs to $\mathscr{U}-\mathscr{F}$. However, it is unknown whether $A \in \mathscr{L}$. Until recently, no other definitive information about the properness of these containments was available.

A reasonable approach to Question 4.5 studies the behavior of these four classes with respect to basic group theoretic operations of taking a free product (Free), a split amalgamated free product (SplitAmalg), and a split HNN extension (SplitHNN) Howie has done this for the two classes $\mathscr{F}$ and $\mathscr{U}$. We summarize his results for these two classes in table (4.1). Each row corresponds to an operation; each column corresponds to one of the classes. Each entry (yes/no) indicates whether the given class is closed under the corresponding operation.

$\begin{array}{ccc} & \mathscr{F} & \mathscr{U} \\ \text { Free } & \text { Yes } & \text { Yes } \\ \text { SplitAmalg } & \text { Yes Yes } \\ \text { SplitHNN } & \text { No Yes }\end{array}$

Adams's group, $A$, can be presented:

$$
A=\left\langle y, x, t \mid y=[y, x], x=y^{t}\right\rangle
$$

so that $A$ is a split HNN extension of the group

$$
\langle y, x \mid y=[y, x]\rangle=\left\langle y, x \mid y^{2}=y^{x}\right\rangle
$$

and the latter is a split HNN extension of $\mathbb{Z}$. $\operatorname{But} \operatorname{ncl}(y, A)$ is a nontrivial perfect subgroup of $A$ so $\mathscr{F}$ is not closed under the operation of taking split HNN extensions. As $\mathscr{U}$ is closed in this respect, $A \in \mathscr{U}-\mathscr{F}$.

Consider the group

$$
G=\left\langle y, t \mid y=\left[y, y^{\left(y^{t}\right)}\right]\right\rangle .
$$

In our introduction we indicated why $G \notin \mathscr{A}$ and how $G$ is a split HNN extension of $A$ and, thus, is the result of a finite number of split HHN extensions of $\mathbb{Z}$. So, neither $\mathscr{L}$ nor $\mathscr{A}$ is closed under split HNN extensions. But $\mathscr{U}$ is closed with respect to this operation. We summarize this in row 3 of table (4.3).

We show below that $\mathscr{A}$ is closed under split amalagamated products. A similar theorem for $\mathscr{L}$ is unresolved. The difficulty highlights the limit of our understanding of the relationship between the geometry of crumpled laminations and the structure of the fundamental groups of the involved manifolds.

All classes are closed under free products. To summarize,

$\begin{array}{ccccc} & \mathscr{F} & \mathscr{L} & \mathscr{A} & \mathscr{U} \\ \text { Free } & \text { Yes } & \text { Yes } & \text { Yes } & \text { Yes } \\ \text { SplitAmalg } & \text { Yes } & ? & \text { Yes } & \text { Yes } \\ \text { SplitHNN } & \text { No } & \text { No } & \text { No } & \text { Yes }\end{array}$


The following lemma holds for both $\mathscr{L}$ and $\mathscr{A}$. For $\mathscr{L}$, we let $P=\operatorname{ker}\left(i_{\#}\right)$ where $i: M \mapsto W$. For $\mathscr{A}$, we let $P=\operatorname{ncl}(\phi(H), G)$. In both cases $P$ is a perfect normal subgroup of $G$.

Lemma 4.6. Suppose $G \notin \mathscr{L}($ resp. $\mathscr{A})$ and $\psi: G \mapsto G_{1}$ is a homomorphism so that $\psi(P) \neq 1 \in G_{1}$. Then $G_{1} \notin \mathscr{L}($ resp. $\mathscr{A})$.

Proof. We consider the two cases separately. First, we have

$$
1 \neq \psi(P)=\psi(\operatorname{ncl}(\phi(H), G))<\operatorname{ncl}\left(\psi \circ \phi(H), G_{1}\right) .
$$

Thus, $\psi \circ \phi(H) \neq 1$. Similarly,

$$
\psi \circ \phi(H)<\psi(\operatorname{ncl}(\phi(\operatorname{Wild}(H))), G)<\operatorname{ncl}\left(\psi \circ \phi(\operatorname{Wild}(H)), G_{1}\right)
$$

implying that $G_{1} \notin \mathscr{A}$, finishing the first case.

Since $\mathscr{L}$ is not as yet understood in group theoretic terms, the proof is rather geometric. Let $(W, M, N)$ be a nontrivial crumpled lamination with $\pi_{1}(M)=G$ and $\psi: G \mapsto G_{1}$ a surjection with $\psi(P) \neq 1$. Let $p: W \mapsto[0,1]$ be the decomposition map. Let $B^{2}=[0,1] \times[0,1], t B^{2}=[0, t] \times[0, t]$, and $W^{\prime}=W \times[0,2] \times[0,2]$. For $0 \leq t \leq 1$ define

$$
h_{t}=\left(p^{-1}(t) \times(1+t) B^{2}\right) \cup\left(p^{-1}([0, t]) \times \operatorname{bdy}\left((1+t) B^{2}\right)\right)
$$

and $q^{\prime}: W^{\prime} \mapsto[0,1]$ by $q\left(h_{t}\right)=t$. Double $W^{\prime}$ along $M \times \operatorname{clos}\left(2 B^{2}-B^{2}\right)$. The map $q^{\prime}$ naturally doubles to a map $q^{\prime \prime}$ from the double to $[0,1]$. This yields a cobordism $\left(W^{\prime \prime}, M^{\prime \prime}, N^{\prime \prime}\right)$ and a crumpled lamination $q^{\prime \prime}: W^{\prime \prime} \mapsto[0,1]$ with

$$
\begin{gathered}
\operatorname{dim}\left(W^{\prime \prime}\right)=n+3, \\
M^{\prime \prime}=q^{\prime \prime-1}(0) \cong M \times S^{2}, \\
N^{\prime \prime}=q^{\prime \prime-1}(1) \cong N \times S^{2} .
\end{gathered}
$$

The point of this construction is that there is a flat embedding of $M \times B^{2} \times[0,1]$ in $W^{\prime \prime}$ with $q^{\prime \prime}\left(M \times B^{2} \times t\right)=t$. There are a finite number of 2-handles which may be attached to $M$ to realize the surjective homomorphism $\psi$. These attachments naturally extend to $M \times B^{2} \times[0,1]$ to yield a crumpled lamination $\left(W_{1}, M_{1}, N_{1}\right)$ with $\pi_{1}\left(M_{1}\right) \cong G_{1}$. Since $\psi(P) \neq 1 \in G_{1}$, this crumpled lamination is nontrivial, and, thus, $G_{1} \notin \mathscr{L}$. This completes the proof of the lemma.

Theorem 4.7. The class $\mathscr{A}$ is closed under the operation of taking split-amalgamated products.

Proof. Suppose $G_{1}, G_{2} \in \mathscr{A}, G=G_{1} *_{K} G_{2}$, and $r: G_{1} \mapsto K$ is the splitting retraction homomorphism. Then $r$ extends to a retraction $s: G \mapsto G_{2}$. If $G \notin \mathscr{L}$ (resp. $\mathscr{A}$ ), then necessarily $1 \neq \phi(H)<P<\operatorname{ker}(s)$ or else $G_{2} \notin \mathscr{L}$ (resp. $\mathscr{A}$ ). Any standard subgroup theorem for a free product with amalgamation (e.g., [LS]) gives the structure of $\phi(H)$ as the free product $F *$ $\left(\Pi\left(\phi(H) \cap G_{1}^{\lambda}\right)\right)$ where $F$ is a free group and $\lambda$ ranges over a subset of $G$. Let $\rho_{\lambda}: \phi(H) \mapsto \phi(H) \cap G_{1}^{\lambda}$ be the natural retraction. Since $G_{1}^{\lambda} \cong G_{1} \in \mathscr{A}$, then $\phi(H) \cap G_{1}^{\lambda}=1$. So, $\phi(H)=F$. But $\phi(\operatorname{Wild}(H))<\operatorname{Wild}(\phi(H))=1$. Thus, $\phi(H)=1$ and $G \in \mathscr{A}$.

The proof of Theorem 4.7 yields 
Corollary 4.8. The class $\mathscr{L}$ is closed under the operation of taking doubly split amalgamated products.

Proof. There is also a retraction $s^{\prime}: G \mapsto G_{1}$. The subgroup theorem then puts the perfect group $P$ in a free subgroup of $G_{1} *_{K} G_{2}$, forcing $P$ to be trivial, and the conclusion follows.

Corollary 4.9. Both $\mathscr{L}$ and $\mathscr{A}$ are closed under free products.

\section{APPENDIX: GeNERALIZED $n$-MANIFOLds}

We examine the construction of $\S 1$ more closely. Let $M$ be an orientable $n$-manifold $(n \geq 5), K \subset \operatorname{int}(M)$ be a tamely embedded almost acyclic 2-complex, and $L$ be a manifold mapping cylinder neighborhood of $K$ in $\operatorname{int}(M)$. All homology and cohomology is with $\mathbb{Z}$ coefficients. We denote the direct sum of $s$ copies of $\mathbb{Z}$ by $\mathbb{Z}^{s}$. By definition we have, for some nonnegative integer, $s$,

$$
H_{q}(L) \cong H_{q}(K)=\left\{\begin{array}{cc}
0, & q \geq 2, \\
\mathbb{Z}^{s}, & q=1, \\
\mathbb{Z}, & q=0 .
\end{array}\right.
$$

We also have the following equivalences:

$$
H_{q}(M, M-K) \cong H_{q}(L, L-K) \cong H_{q}(L, \operatorname{bdy}(L)) \cong H_{n-q}(L) .
$$

The first is by excision, the second by regular neighborhood theory, and the last by duality. So,

$$
H_{q}(M, M-K) \cong \begin{cases}0, & q \leq n-2 \text { or } q>n, \\ \mathbb{Z}^{s}, & q=n-1, \\ \mathbb{Z}, & q=n .\end{cases}
$$

Consider the exact sequence of $(L, \operatorname{bdy}(L))$ :

(A.4)

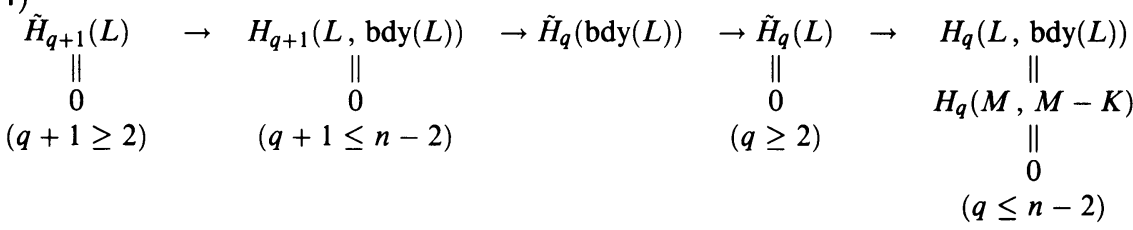

Inspection yields

$$
\tilde{H}_{q}(\operatorname{bdy}(L)) \cong \begin{cases}\mathbb{Z}, & q=n-1 \\ \mathbb{Z}^{s}, & q=n-2 \\ \mathbb{Z}^{s}, & q=1, \\ 0, & \text { otherwise }\end{cases}
$$

as long as $n \geq 4$.

Consider the identification spaces $L / K$ and $M / K$ and let $x=|K|$. Obviously, $L / K$ is an AR and has trivial reduced homology. $M / K$ is an ANR; it follows from the exact sequence of $(L / K, L / K-x)$ that $H_{q}(L / K, L / K-x) \cong$ $\tilde{H}_{q-1}(L / K-x)$ and from regular neighborhood theory that the latter is isomorphic to $\tilde{H}_{q-1}(\operatorname{bdy}(L))$. This and excision yield

$$
H_{q}(M / K, M / K-x) \cong \tilde{H}_{q-1}(\operatorname{bdy}(L)) .
$$


The grope construction of $\S 1$ produces a cell-like map, $d:(Y, y) \mapsto(M / K, x)$, where $W=d^{-1}(x)$ is a wedge of arcs with wedgepoint $y, Y$ is an ANR, and and $Y-y$ is a manifold. In the manifold, $Y-y$, duality gives

$$
H_{q}(Y-y, Y-W) \cong H_{c}^{n-q}(W-y)=0
$$

so the homology sequence of the triple $(Y, Y-y, Y-W)$ shows that

$$
H_{q}(Y, Y-W) \cong H_{q}(Y, Y-y) \text {. }
$$

But $d:(Y, Y-W) \mapsto(M / K, M / K-x)$ is a homology equivalence so (A.5), (A.6), and (A.8) imply

$$
H_{q}(Y, Y-y) \cong \tilde{H}_{q-1}(\operatorname{bdy}(L)) \cong \begin{cases}\mathbb{Z}, & q=n \\ \mathbb{Z}^{s}, & q=n-1, \\ \mathbb{Z}^{s}, & q=2, \\ 0, & \text { otherwise }\end{cases}
$$

The construction of $\S 1$ also identifies a wedge of 2-spheres, $S=\bigvee_{j=1}^{s} S_{j}^{2}$ $\subset Y$ with wedgepoint $y$, with each $S_{j}^{2}$ representing a distinct generator of $\mathrm{H}_{2}(Y, Y-y)$. Inspection of the following diagram shows that the homology sequence of $(Y, Y-y)$ splits at $H_{2}(Y)$ : (A.10)

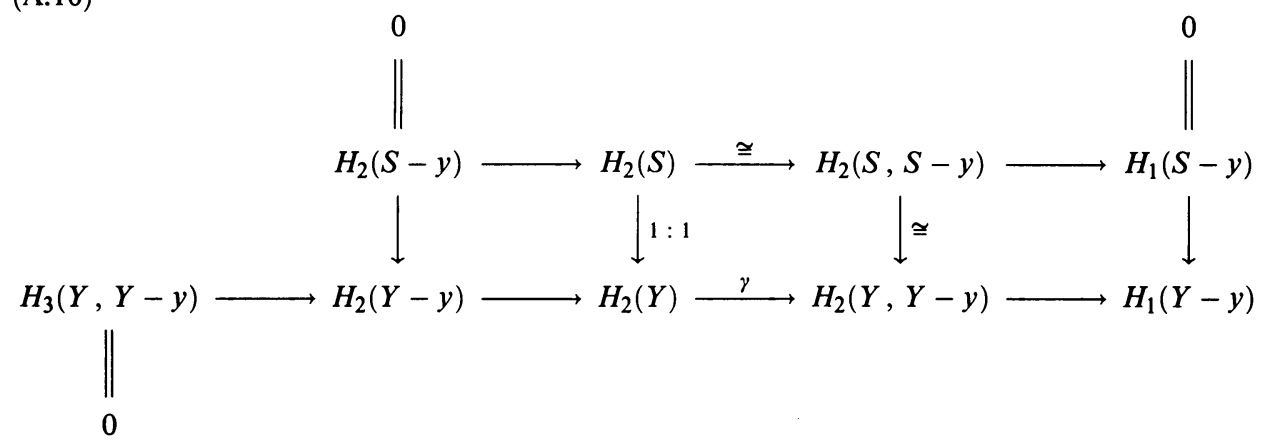

Thus, (A.9) and (A.10) force

$$
H_{2}(Y) \cong H_{2}(Y-y) \oplus H_{2}(Y, Y-y) \cong H_{2}(Y-y) \oplus \mathbb{Z}^{s} \text {. }
$$

Let $\delta: Y \mapsto Y / S$ be the identification map and let $Z=Y / S$ and $z=|S|$. The goal of this appendix is to show that $Z$ is a homology manifold, i.e.,

$$
H_{q}(Z, Z-z) \cong \begin{cases}\mathbb{Z}, & q=n, \\ 0, & q \neq n .\end{cases}
$$

First, note that $\tilde{H}_{q}(Z)=\tilde{H}_{q}(Y / S) \cong H_{q}(Y, S)$. The exact homology sequence for the pair, $(Y, S)$ yields $H_{q}(Y) \cong H_{q}(Y, S) \quad(q \neq 2)$. For $q=2$ we have shown (A.10) that the inclusion $S \mapsto Y$ induces a splitting at the level of second homology so

$$
H_{2}(Y) \cong H_{2}(S) \oplus H_{2}(Y, S) \cong \mathbb{Z}^{s} \oplus H_{2}(Y, S)
$$

and (A.11) and finitely generated abelian group theory show

$$
H_{2}(Y, S) \cong H_{2}(Y-S) \text {. }
$$

Now, decompose $S$ as $S=Q_{1} \cup Q_{2}$ where $Q_{1}$ is a wedge of $s$ closed 2-disks, $Q_{2}$ is a disjoint union of $s$ closed 2-disks, and $Q_{1} \cap Q_{2}$ is a disjoint union 
of $s$ simple closed curves. Duality applies [Do, Chapter VIII, $\S 7]$ to give the Mayer-Vietoris sequence

$$
H_{q}(Y, Y-S) \rightarrow H_{q}\left(Y, Y-Q_{1}\right) \oplus H_{q}\left(Y, Y-Q_{2}\right) \rightarrow H_{q}\left(Y, Y-Q_{1} \cap Q_{2}\right) \rightarrow
$$

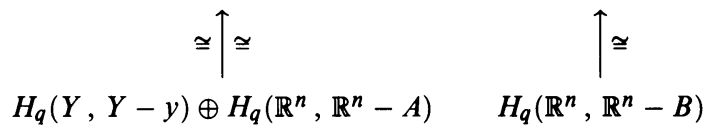

where $A$ and $B$ are finite sets of $s$ points and simple closed curves respectively. Then (A.9) and a diagram chase show that

$$
H_{q}(Y, Y-S) \cong\left\{\begin{array}{cc}
\mathbb{Z}, & q=n, \\
\mathbb{Z}^{s}, & q=2, \\
0, & \text { otherwise. }
\end{array}\right.
$$

Finally, $\delta:(Y, Y-S) \mapsto(Z, Z-z)$ induces the diagram

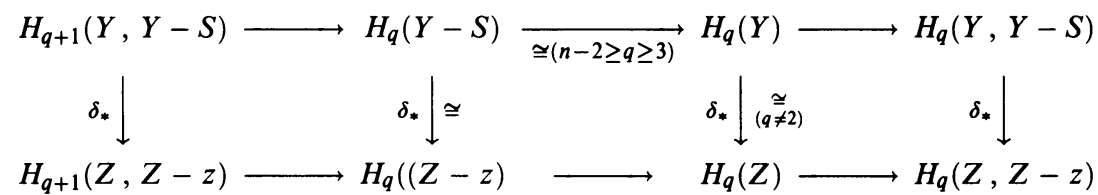

It is immediate that

$$
H_{q}(Z, Z-z) \cong \begin{cases}\mathbb{Z}, & q=n, \\ 0, & n-2 \geq q \geq 4 .\end{cases}
$$

The cases $q=n-1,3,2$, or 1 follow from careful diagram chases using (A.14) and (A.16).

\section{REFERENCES}

[Ad] J. F. Adams, A new proof of a theorem of W. H. Cockcroft, J. London Math. Soc. 49 (1955), 482-488.

[Ar] S. Armentrout, Decompositions and absolute neighborhood retracts, Geometric Topology (L. C. Glaser and T. B. Rushing, eds.), Lecture Notes in Math., vol. 438, Springer-Verlag, Berlin and New York, 1975, pp. 1-5.

[B] R. H. Bing, Upper semicontinuous decompositions of $E^{3}$, Ann. of Math. (2) 65 (1957), 363-374.

[C1] J. W. Cannon, Shrinking cell-like decompositions of manifolds. Codimension three, Ann. of Math. (2) 110 (1979), 83-112.

[C2] The recognition problem: what is a topological manifold?, Bull. Amer. Math. Soc. 84 (1978), 832-866.

[CBL] J. W. Cannon, J. L. Bryant, and R. C. Lacher, The structure of generalized manifolds having nonmanifold set of trivial dimension, Geometric Topology (J. C. Cantrell, ed.), Academic Press, New York, 1979, pp. 261-300.

[Da1] R. J. Daverman, Every crumpled n-cube is a closed 4-cell-complement, Michigan Math. J. 24 (1977), 225-241.

[Da2] _ _ Detecting the disjoint disks property, Pacific J. Math. 93 (1981), 277-298.

[Da3] _ Decompositions into codimension one submanifolds, Compositio Math. 55 (1985), 185-207.

[Da4] _ Decompositions of manifolds, Academic Press, Orlando, 1986.

[DT1] R. J. Daverman and F. C. Tinsley, Laminated decompositions involving a given submanifold, Topology Appl. 20 (1985), 107-119.

[DT2] __ Laminations, finitely generated perfect groups, and acyclic mappings, Michigan Math. J. 33 (1986), 343-351. 
[DT3] _ The homotopy type of certain laminated manifolds, Proc. Amer. Math. Soc. 96 (1986), 703-708.

[DW1] R. J. Daverman and J. J. Walsh, Decompositions into codimension two spheres and approximate fibrations, Topology Appl. 19 (1985), 103-121.

[DW2] __ Decompositions into codimension two manifolds, Trans. Amer. Math. Soc. 288 (1985), 273-291.

[DW3] _ Decompositions into submanifolds that yield generalized manifolds, Topology Appl. 26 (1987), 143-162.

[Do] Albrecht Dold, Lectures on algebraic topology, Springer-Verlag, Berlin, Heidelberg, and New York, 1972.

[Dr] A. N. Dranishnikov, On a problem of P. S. Aleksandrov, Math. USSR-Sb. 63 (21) (1989), 539-545; English transl. of Mat. Sb. 135 (177) (1988), 551-557.

[E1] R. D. Edwards, Demension theory. I, Geometric Topology (L. C. Glaser and T. B. Rushing, eds.), Lecture Notes in Math., vol. 438, Springer-Verlag, Berlin and New York, 1975, pp. 195-211.

[E2] _ The topology of manifolds and cell-like maps, Proc. Internat. Congr. Math. Helsinki, 1978 (O. Lehti, ed.), Acad. Sci. Fenn., Helsinki, 1980, pp. 111-127.

[F] M. H. Freedman, The topology of four-dimensional manifolds, J. Differential Geom. 17 (1982), 352-453.

[H] J. Howie, Aspherical and acyclic 2-complexes, J. London Math. Soc. (2) 20 (1979), 549-558.

[Li] V. T. Liem, Manifolds accepting codimension one sphere-like decompositions, Topology Appl. 21 (1985), 77-86.

[LS] R. C. Lyndon and P. E. Schupp, Combinatorial group theory, Springer-Verlag, Berlin and New York, 1976.

[Q1] D. Quillen, Cohomology of groups, Actes Congres Int. Math., Tome 2, 1970, pp. 47-51.

[Qn1] F. Quinn, Ends of maps. I, Ann. of Math. (2) 110 (1979), 275-331.

[Qn2] _ Resolutions of manifolds, and the topological characterization of manifolds, Invent. Math. 72 (1983), 267-284.

[Qn3] _ An obstruction to the resolution of homology manifolds, Michigan Math. J. 34 (1987), 285-291.

[Si] L. C. Siebenmann, The obstruction to finding a boundary for an open manifold of dimension greater than 5, Ph.D. thesis, Princeton Univ., 1965.

[Sm] S. Smale, A Vietoris theorem for homotopy, Proc. Amer. Math. Soc. 8 (1957), 604-610.

[T] F. C. Tinsley, Acyclic maps which are homotopic to homeomorphisms, Abstract \#838-57-31, Abstracts Amer. Math. Soc. 8 (1987), p. 426.

[W] C. T. C. Wall (Editor), Homological group theory, Cambridge Univ. Press, London, 1979. 1300

Department of Mathematics, University of Tennessee, Knoxville, Tennessee 37996-

Department of Mathematics, Colorado College, Colorado Springs, Colorado 80903 